\title{
Hermite-Gaussian decomposition of free electron laser optical fields
}

\author{
S. P. Niles, ${ }^{*}$ J. Blau, ${ }^{\dagger}$ and W. B. Colson ${ }^{\ddagger}$ \\ Naval Postgraduate School, Monterey, California 93940, USA
}

(Received 22 October 2009; published 30 March 2010)

\begin{abstract}
Free electron lasers (FELs) are favored in many research applications due to their ability to operate primarily in the fundamental (Gaussian) mode. In oscillator FELs, the appropriate basis set for decomposition is uniquely determined by the cavity parameters. Since FEL amplifiers generate and amplify the optical field over the length of the undulator influenced only by the electron beam, the appropriate basis set is not uniquely determined. A methodology is presented to decompose the optical fields with a Hermite-Gaussian basis set chosen such that the number of higher-order modes is minimized. The methodology is used to study amplifier FEL optical beams for comparison with experiment.
\end{abstract}

\section{INTRODUCTION}

Applications requiring excellent beam quality and propagation characteristics lend themselves to using free electron lasers (FELs) as their light sources. Laboratory users need accurate representations of the optical modes they are using. The output light from an oscillator FEL can be strongly affected by the cavity parameters, and the light fields can be decomposed via a two-dimensional HermiteGaussian (HG) basis set [1] with well-defined characteristics determined by the wavelength, the mirror radii of curvature, and the distance between the mirrors. In an amplifier, there is no cavity to determine the basis set. Even with a seeding light source, the large single pass amplification and guiding experienced as the light passes through the undulator of an amplifier results in virtually no residual of the seed laser's optical modes remaining after the gain region.

The HG polynomials serve as a solution to the wave equation and form an orthonormal basis set with which to expand any electromagnetic field of interest. We start from a dimensionless paraxial wave equation, where we have already applied the slowly varying amplitude and phase approximation [1],

$$
\left[-\frac{l}{4} \tilde{\nabla}_{\perp}^{2}+\frac{\partial}{\partial \tau}\right] a(\overrightarrow{\widetilde{r}}, \tau)=0
$$

where $a$ is the complex, dimensionless optical field [2] proportional to the electric field, $\tau$ is the dimensionless time normalized to the time required for a photon to traverse the undulator $(\tau=c t / L)$, and $\tilde{\nabla}_{\perp}^{2}$ is the dimensionless Laplacian operator,

$$
\tilde{\nabla}_{\perp}^{2}=\frac{\partial^{2}}{\partial \tilde{x}^{2}}+\frac{\partial^{2}}{\partial \tilde{y}^{2}}=\frac{2 L}{k}\left(\frac{\partial^{2}}{\partial x^{2}}+\frac{\partial^{2}}{\partial y^{2}}\right),
$$

\footnotetext{
*spniles@nps.edu

blau@nps.edu

‡wbcolson@nps.edu
}

where $L$ is the undulator length, $k$ is the wave number of the propagating field, and dimensionless transverse coordinates are defined by $\tilde{x}=x / \sqrt{2 L / k}$ and $\tilde{y}=y / \sqrt{2 L / k}$. We can proceed to solve the wave equation outside the gain medium [2], arriving at

$$
a_{m, n}(\vec{r}, \tau)=\frac{a_{0} w_{0}}{w} H_{m}\left(\frac{x \sqrt{2}}{w}\right) H_{n}\left(\frac{y \sqrt{2}}{w}\right) \mathrm{e}^{-\left(r^{2} / w^{2}\right)} \mathrm{e}^{l \phi_{m, n}(\vec{r}, \tau)},
$$

where $a_{m, n}(\vec{r}, \tau)$ is the solution to Eq. (1) for the $m, n$ mode where all terms are dimensionless (and tildes have now been dropped for ease of reading), $a_{0}$ is the field amplitude at the center of the field at the mode waist $(\tau=0), w_{0}$ is the mode radius at the waist, $w$ is the mode radius at a particular time $\tau$ given by $w=\sqrt{z_{0}+\left(\tau / z_{0}\right)^{2}}, z_{0}$ is the normalized Rayleigh length $\left(z_{0}=Z_{R} / L, \quad Z_{R}\right.$ is the dimensional Rayleigh length), $H_{m}$ is the value of the $m$ th order Hermite-Gaussian polynomial, $r^{2}=x^{2}+y^{2}$, and the phase $\phi_{m, n}(\vec{r}, \tau)$ is given by

$$
\phi_{m, n}(\vec{r}, \tau)=\frac{r^{2} \tau}{w_{0}^{2} w^{2}}-(m+n+1) \tan ^{-1}\left(\frac{\tau}{z_{0}}\right) .
$$

If we then look at characteristics of Eqs. (2) and (3), we can substitute $\Omega \equiv \tau / z_{0}$ as a measure of where the field is in its propagation path, defining the phase relationship, and we can define a characteristic area, $\Lambda \equiv \pi w^{2}=\pi z_{0}(1+$ $\left.\tau^{2} / z_{0}^{2}\right)$, which reflects the size of the mode. Making these substitutions, we arrive at

$$
\begin{aligned}
a_{m, n}(\vec{r}, \Omega)= & a_{0} \sqrt{\frac{1}{1+\Omega^{2}}} H_{m}\left(x \sqrt{\frac{2 \pi}{\Lambda}}\right) H_{n}\left(y \sqrt{\frac{2 \pi}{\Lambda}}\right) \\
& \times \mathrm{e}^{-\left(\pi r^{2} / \Lambda\right)} \mathrm{e}^{i \phi_{m, n}(\vec{r}, \Omega)}
\end{aligned}
$$

and

$$
\phi_{m, n}(\vec{r}, \Omega)=\frac{\pi r^{2} \Omega}{\Lambda}-(m+n+1) \tan ^{-1}(\Omega) .
$$


We see from our final equations, (4) and (5), that $\Lambda$ acts as a transverse scaling factor in the basis, altering how the Hermite polynomials grow in size and how the Gaussian portion quashes them away from the center of the field. This scaling of the basis set is a critical parameter that will be addressed in the next section.

It is important to note that there is no unique choice of basis scaling factors for a given field - there are an infinite number of solutions [3] resulting in different mode decompositions for the same field, each equally valid. Other methods have been proposed [4], and discuss the basis scaling factors, but never directly address the methodology employed to fix the two free parameters. The choice of scaling factors is decoupled from the propagation mechanism and determined solely by minimizing the number of modes necessary to decompose the optical field to a preselected tolerance. This decomposition method is intended as a decomposition of the optical field, as a diagnostic, after it has left the interaction region as a way to compare with other types of lasers for mode content/propagation characteristics on an equal footing. This has not been discussed in previous papers.

\section{METHODOLOGY}

Using the Naval Postgraduate School (NPS) 4D FEL amplifier simulation [5], the appropriate parameters to model an existing or proposed FEL result in an output field at an arbitrary point beyond the undulator. The NPS 4D FEL code represents the optical field in terms of wavefronts (real and imaginary parts of the complex optical field

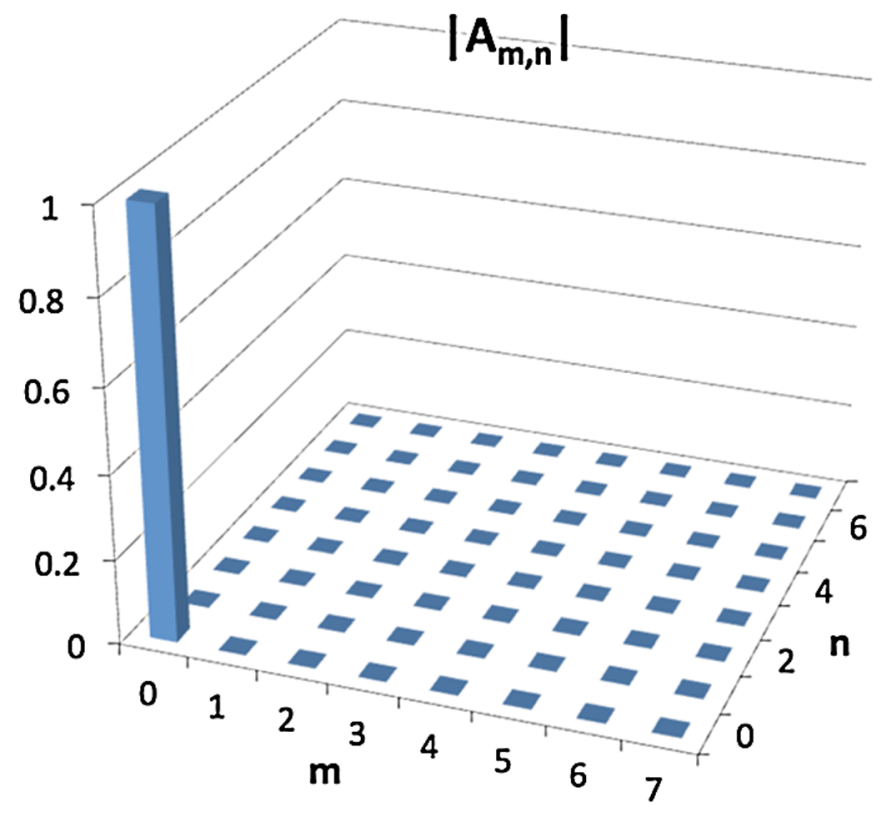

at each grid point), so it is necessary to choose a suitable basis set in order to determine the optical mode content. This optical field is first normalized in intensity and then an iterative process is used to search the scaling area and phase parameter space for the best fit parameters. The first step is to analyze the first two moments of the intensity field.

The first moment provides the center of intensity, from which a radius $R$ is measured. This is calculated (using the $x$ coordinate as an example) through

$$
\bar{x}=\frac{\int x|a(x, y)|^{2} d x}{\int|a(x, y)|^{2} d x} .
$$

We choose to take the second moment to provide an upper bound on the values of $\Lambda$. For higher-order modes, the intensity is less concentrated in the central region and the Gaussian mode (fundamental) necessary to obtain a similar second moment is larger, resulting in a nonideal scaling factor. To determine the second moment, we calculate

$$
R^{2}=2 \frac{\iint|a(x, y)|^{2}\left[(x-\bar{x})^{2}+(y-\bar{y})^{2}\right] d x d y}{\iint|a(x, y)|^{2} d x d y} .
$$

The field is decomposed by projecting onto the HG basis modes for a specific $\Lambda$,

$$
\begin{aligned}
A_{m, n}= & \frac{a_{0}}{\sqrt{1+\Omega^{2}}} \frac{1}{\sqrt{2^{m} 2^{n} m ! n !}} H_{m}\left(x \sqrt{\frac{2 \pi}{\Lambda}}\right) H_{n}\left(y \sqrt{\frac{2 \pi}{\Lambda}}\right) \\
& \times \mathrm{e}^{-\imath \phi_{m, n}},
\end{aligned}
$$

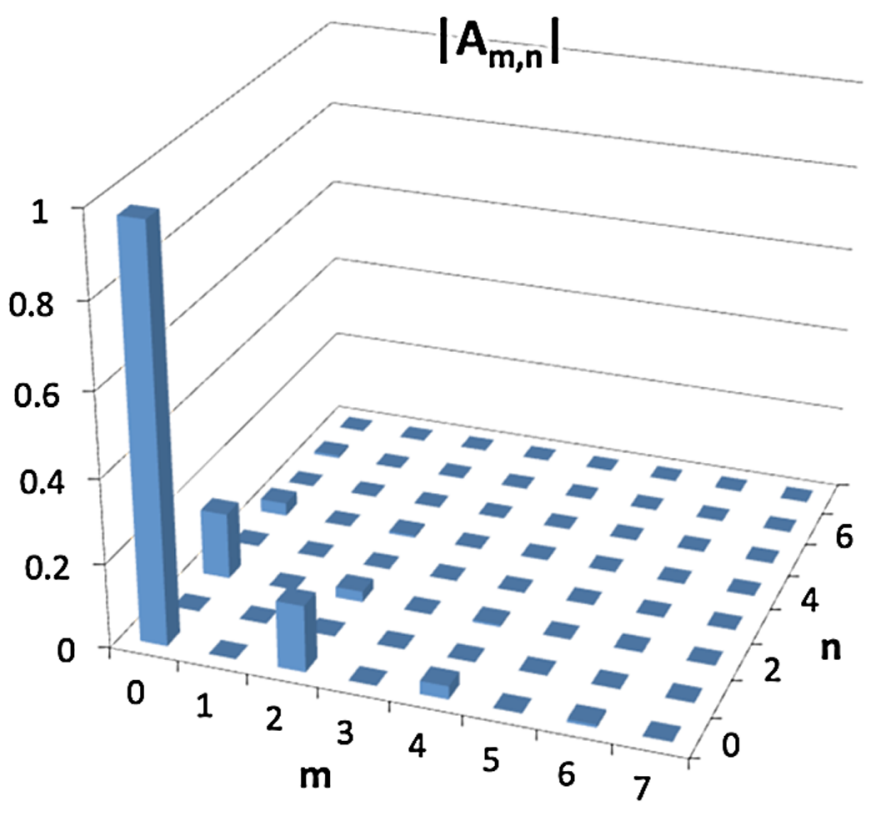

FIG. 1. (Color) Modal composition histogram of a purely $(0,0)$ mode (coefficient area $=1.0$ ) against the correct basis, $\Omega=1.25$ and $\Lambda=4.0252$ (left) and a different scaling factor, $\Lambda=3.0$, resulting in a coefficient area $=1.06$ and finding $94.7 \%$ in the $(0,0)$ mode and $\approx 2.5 \%$ in the $(0,2)$ and $(2,0)$ modes (right). Histograms are plotted as $\left|C_{m, n}\right|$ to better show the higher-order modes, using the color scale shown on the right. 
where all coefficients $A_{m, n}$ are normalized. The contribution of a particular $(m, n)$ mode is captured in the complex coefficients,

$$
C_{m, n}=\int_{-\infty}^{\infty} \int_{-\infty}^{\infty} A_{m, n}^{*}(x, y) a(x, y) d x d y
$$

where $a(x, y)$ is the field being analyzed and $A_{m, n}^{*}$ is the complex conjugate of the $(m, n)$ basis function. We choose the scaling factor that minimizes the number of modes necessary to decompose the field of interest, which bounds $\Lambda$ to the interval $0<\Lambda \leq \pi R^{2}$ from Eq. (7).

To minimize the number of modes present in a decomposition, the peak value, $\left|C_{m, n}\right|^{2}$, within the coefficient space is found and used to normalize the remaining coefficients (thus, the peak $\left|C_{m, n}\right|^{2}$ is always equal to 1.0). The values for that particular choice of $\Lambda$ and $\Omega$ are summed, generating a "coefficient area," and compared to other basis choices. The basis set with the smaller "coefficient area" is chosen as "better," as it contains fewer contributing modes. In the case of a single mode $(0,0)$, or pure Gaussian field, the choice of $\Lambda$ that corresponds to the scaling factor that generated the field would result in a single mode populated with a coefficient area of 1.0. A choice departing from the $\Lambda$ that was used to generate the field would result in higher-order modes being populated when it is decomposed against the improperly scaled basis set. Figure 1 demonstrates this effect. The topology of this coefficient area (see Fig. 2) approach can be somewhat complicated; however for nearly Gaussian modes, as are typically found in FELs, the optimal basis scaling factor can be determined fairly quickly, as it lies at the bottom of the central valley.

To validate the choice of basis scaling factor, $\Lambda$, and phase relationship, $\Omega$, the two-dimensional cross correlation is used as a figure of merit. First, a test field, $T$, is generated from the $C_{m, n}$ 's and normalized in intensity.

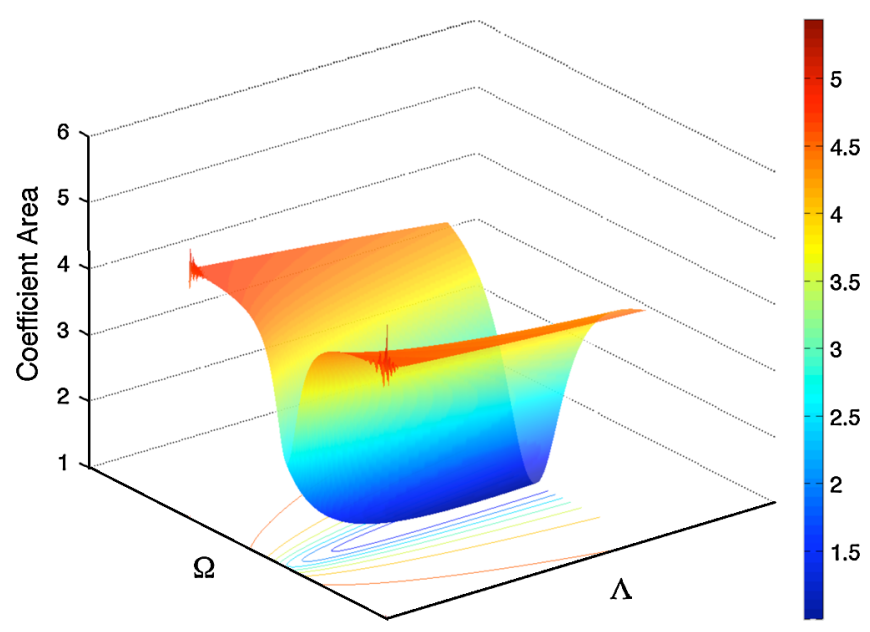

FIG. 2. (Color) Topology of the coefficient area map for a purely $(0,0)$ mode with $\Lambda=4.0252$ and $\Omega=1.25$ over the ranges $-3 \pi \leq \Omega \leq 3 \pi$ and $0.1 \times \pi R^{2} \leq \Lambda \leq \pi R^{2}$.
Since $a$ and $T$ are both intensity normalized, if $T$ is an exact copy of $a$, the result of this cross correlation is an autocorrelation and the resulting array will have a maximum value of 1.0 at the position where the generated field overlaps the actual field exactly.

\section{SIMULATION RESULTS}

The FEL research group at the Naval Postgraduate School has performed simulations of numerous existing FELs and provided supporting simulation work for many more proposed designs. Key parameters for the designs analyzed in [5] are summarized in Table I. These FELs were simulated and the optical field analyzed upon exit from the undulator to determine their mode content.

TABLE I. FEL amplifier parameters, where $N$ is the number of undulator periods, $\lambda_{0}$ is the length of a single undulator period, $K_{\mathrm{rms}}$ is the undulator parameter, $E$ is the beam energy, $q$ is the bunch charge, $t_{b}$ is the bunch duration, and $\Delta B / B$ is the undulator taper rate.

\begin{tabular}{lcccc}
\hline \hline & $\begin{array}{c}\text { Brookhaven } \\
\text { SDL [6] }\end{array}$ & $\begin{array}{c}\text { Brookhaven } \\
\text { proposed } \\
{[7]}\end{array}$ & $\begin{array}{c}\text { Los Alamos } \\
\text { proposed [8] } \\
\text { step taper }\end{array}$ & $\begin{array}{c}\text { Los Alamos } \\
\text { proposed [8] } \\
\text { linear taper }\end{array}$ \\
\hline$N$ & 256 & 120 & 110 & 110 \\
$\lambda_{0}$ & $3.9 \mathrm{~cm}$ & $3.275 \mathrm{~cm}$ & $2.18 \mathrm{~cm}$ & $2.18 \mathrm{~cm}$ \\
$K_{\mathrm{rms}}$ & 0.78 & 0.7 & 1.2 & 1.2 \\
$E$ & $102 \mathrm{MeV}$ & $80 \mathrm{MeV}$ & $81 \mathrm{MeV}$ & $81 \mathrm{MeV}$ \\
$q$ & $0.35 \mathrm{nC}$ & $1.4 \mathrm{nC}$ & $1 \mathrm{nC}$ & $1 \mathrm{nC}$ \\
$t_{b}$ & $1 \mathrm{ps}$ & $2.8 \mathrm{ps}$ & $1 \mathrm{ps}$ & $1 \mathrm{ps}$ \\
$\frac{\Delta B}{B}$ & $-5 \%$ & $0 \%$ & $-2.5 \%$ & $-8.0 \%$ \\
\hline \hline
\end{tabular}

TABLE II. Modal composition as found by minimizing the coefficient area of the optical mode captured at the undulator exit. Cross-correlation value is reported as the figure of merit (FOM). (* used $14 \times 14=196$ modes to perform decomposition).

\begin{tabular}{lccc}
\hline \hline & Modes & \% Composition & FOM \\
\hline BNL SDL* & $(0,0)$ & $32.3 \%$ & 0.983 \\
& $(2,0)$ & $15.0 \%$ & \\
& $(0,2)$ & $15.0 \%$ & \\
& $(4,0)$ & $6.4 \%$ & \\
BNL proposed & $(0,4)$ & $6.3 \%$ & 0.996 \\
& $(0,0)$ & $95.3 \%$ & \\
& $(2,2)$ & $1.0 \%$ & \\
LANL step & $(0,4)$ & $1.3 \%$ & 0.991 \\
LANL linear & $(4,0)$ & $1.3 \%$ & 0.987 \\
& $(0,0)$ & $99.4 \%$ & \\
& $(0,0)$ & $94.4 \%$ & \\
& $(0,2)$ & $1.5 \%$ & \\
& $(2,0)$ & $1.4 \%$ & \\
& $(0,4)$ & $1 \%$ & \\
& $(4,0)$ & $1 \%$ & \\
&
\end{tabular}




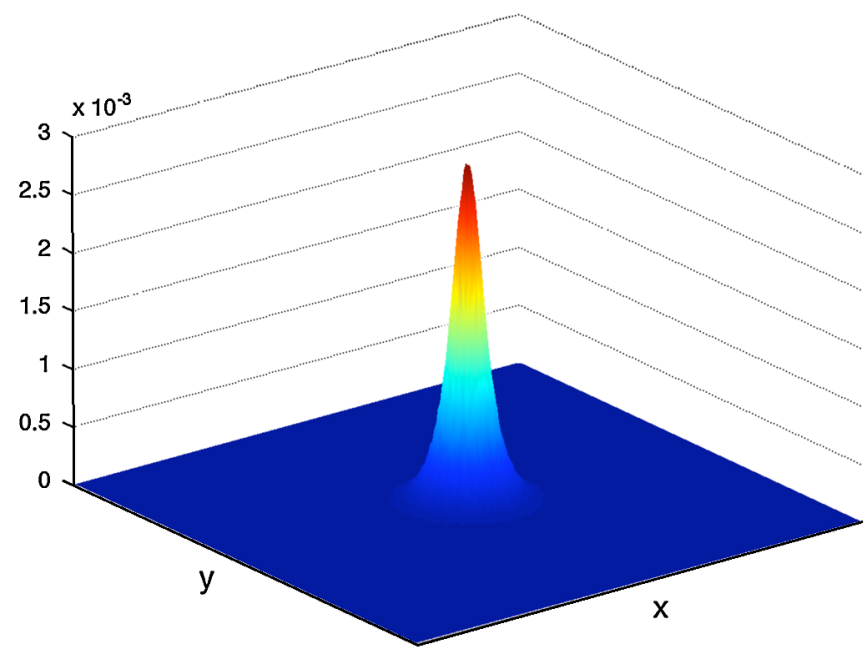

FIG. 3. (Color) Normalized $\left(\left|\iint a(x, y)\right|^{2} d x d y=1\right)$ intensity of light at the exit of the Brookhaven SDL undulator. Intensity in arbitrary units. Transverse dimension in dimensionless units is $-3.16 \leq x \leq 3.16$ and the same for $y$.

The algorithm previously described was applied to the FELs as listed in Table I including through the 7th order modes. Analysis shows that each of these designs does operate primarily in the fundamental mode. Table II shows the top five modes containing more than $1 \%$ power and the cross-correlation value for the best (smallest) coefficient area found. Figure 3 shows the optical intensity of the Brookhaven SDL and Fig. 4 displays the mode content histogram of mode content using a proposed undulator at Brookhaven.

Obtaining a perfect match (cross-correlation value of $1.0)$ is very difficult in practical application. The optical fields that are generated from the FEL simulation code sometimes show unique fine structure. The decomposition process will introduce higher-order modes in an attempt to account for this, but the necessity to truncate the infinite series results, typically, in a much smoother approximated "guess field." The result of this truncation with the HG basis set is analogous to the resulting Fourier decomposition errors of a square wave when the series approximation is truncated. Additionally, as was seen in the coefficient area maps, the slope of the central valley is very shallow as $\Lambda$ is increased and these similar coefficient areas result in similar mode content. Allowing for additional search time for the algorithm to continue its progress down the valley does not result in significant change in the modes present or their content, especially for the fundamental which was of primary concern for this study.

The only field that presented a possible issue with this algorithm was the Brookhaven SDL FEL. Using only through the $(7,7)$ modes, the algorithm found the mode to be primarily in the fundamental but with only a 0.736 cross-correlation value. Reviewing the simulation results, this particular FEL design experiences a full synchrotron oscillation of trapped electrons resulting in a significant

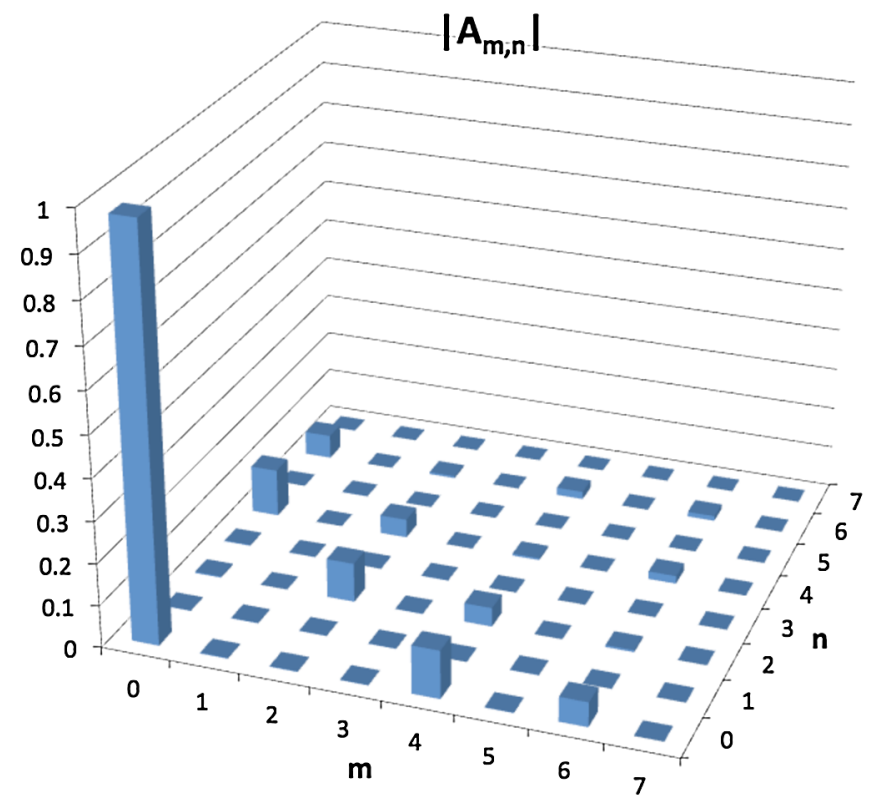

FIG. 4. (Color) Histogram of coefficients contained in the field at the exit of the Brookhaven proposed undulator. The normalized $\left|C_{m, n}\right|^{2}$ shows $95 \%$ of the mode's power is in the $(0,0)$ mode. The $(2,2),(4,0)$, and $(0,4)$ modes contain approximately $1 \%$ of the power each. No other mode contains more than $1 \%$ power through the $(7,7)$ mode.

portion of the power being removed from the central area of the mode prior to tapering. This decrease in central power followed by regeneration results in a "halo" formation that could possibly require significant higher-order modes to properly decompose the field. The simulation was run again, allowing the inclusion of modes through the 13th order (for a total of 196 modes included). The results (shown in Table II) allow for the regeneration of the original mode with significant confidence as the crosscorrelation value has now increased to 0.983 .

If we refer back to Fig. 2 which shows the topology of the ideal coefficient area space, we see that the topology of the LANL linear taper example, shown in Fig. 5, is very similar. Thus we have confidence that the basis set found is representative of a "mostly" Gaussian field. The crosscorrelation value of 0.987 also gives us confidence that the test field generated from the basis set and associated coefficients are representative of the original field.

As an example of a FEL mode that is not as strongly Gaussian, the BNL SDL exhibits a much more complicated coefficient composition. While the cross-correlation value gives us some confidence that the modes obtained generate an excellent facsimile of the original field, we can look at the coefficient area topology, shown in Fig. 6, and immediately see that the topology is much different than a pure Gaussian.

For coefficient maps away from the waist $(\Omega=0)$, we see a skewing of the central valley in the direction of propagation $(\Omega>0$ after the waist and $\Omega<0$ prior to 

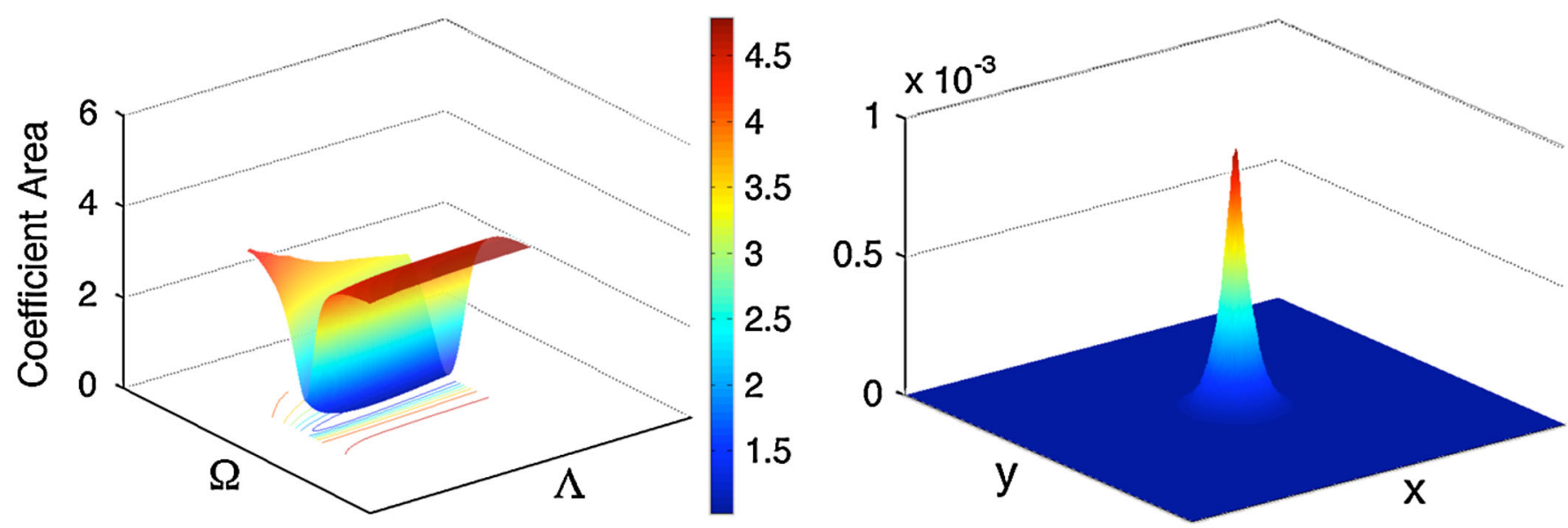

FIG. 5. (Color) The left side is the coefficient area map of the LANL linear taper system with $-3 \pi \leq \Omega \leq 3 \pi$ and $1.0369 \leq \Lambda \leq$ 10.3694. On the right is the normalized light intensity pattern at the undulator exit. Intensity is in arbitrary units. Transverse dimension in dimensionless units is $-6.0302 \leq x \leq 6.0302$ and the same for $y$.
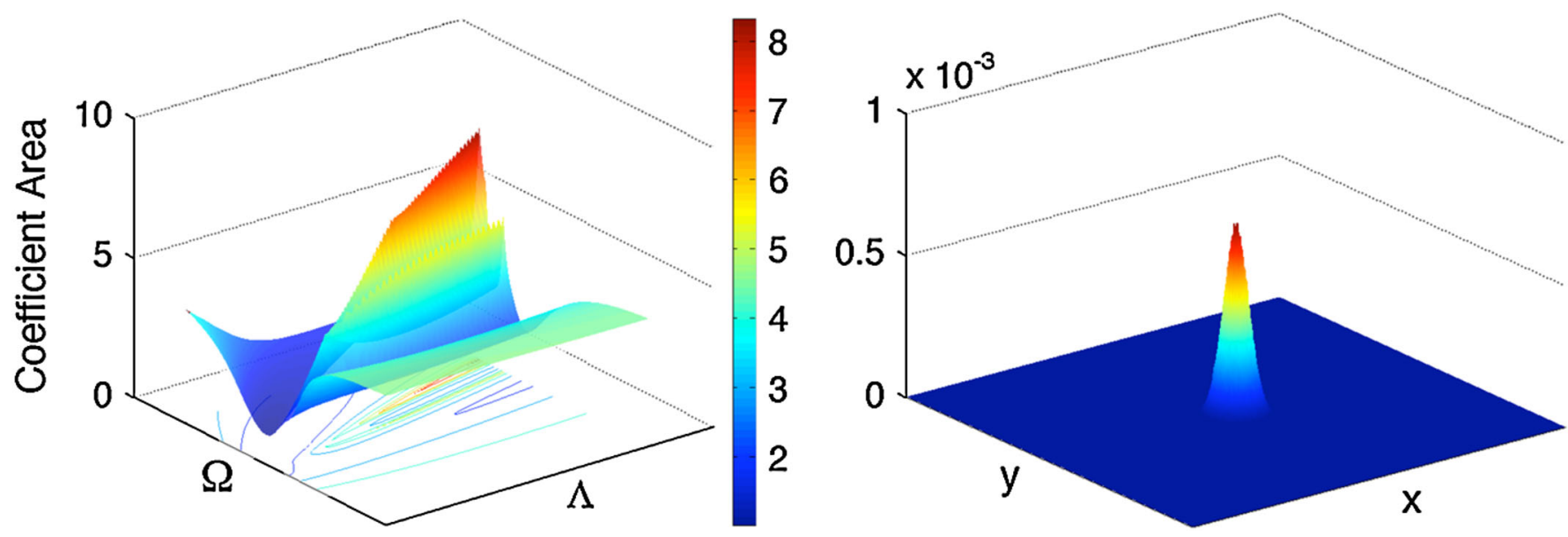

FIG. 6. (Color) The left side is the coefficient area map of the BNL SDL system with $-3 \pi \leq \Omega \leq 3 \pi$ and $1.0369 \leq \Lambda \leq 10.3694$. On the right is the normalized light intensity pattern at the undulator exit. Intensity is in arbitrary units. Transverse dimension in dimensionless units is $-5.163 \leq x \leq 5.163$ and the same for $y$.

the waist). Figure 6 shows both a skew (as expected since this is after the mode's presumed waist) and significant deformation of the central valley. The development of this "hump" is an indicator that the mode being analyzed has significant mode content outside the fundamental.

As seen from these simulations, FEL amplifiers appear to operate primarily in the fundamental mode. We have also shown that this technique allows for the decomposition of more complicated mode combinations that might result from a less than optimal FEL design. Having a modal decomposition module for FEL simulations allows for experimentalists to obtain an excellent prediction of the mode content a particular design can be expected to achieve. It allows for the designers to include more realistic beam mode content as a design criteria and provides them a tool for exploring the final output beam.

\section{ACKNOWLEDGMENTS}

This work was supported by the Office of Naval Research and the High Energy Laser Joint Technology Office.

[1] A.E. Siegman and E. A. Szilas, Appl. Opt. 13, 2775 (1974).

[2] R. Vigil, Master's thesis, Naval Postgraduate School, 2006.

[3] A.E. Siegman, Lasers (University Science Books, Sausalito, CA, 1986).

[4] P. Sprangle, H.P. Freund, B. Hafizi, and J. R. Peñano, Quantum Electron. 45, 218 (2009).

[5] J. Blau, D. Burgraff, T. Y. Voughs, and W. B. Colson, in Proceedings of the 28th Free Electron Laser Conference, 
Berlin, Germany, 2006, p. 222.

[6] T. Watanabe et al., in Proceedings of the 27th Free Electron Laser Conference, Stanford, California, 2005, p. 526.

[7] I. Ben-Zvi, D. Kayran, and V. Litvinenko, in Proceedings of the 27th Free Electron Laser Conference, Stanford, California, 2005, p. 232.

[8] D. C. Nguyen, W. B. Colson, and H. Freund, in Proceedings of the 28th Free Electron Laser Conference, Berlin, Germany, 2006, p. 509. 